\title{
Análise de categorias conceituais da CDD intermediada pela terminologia
}

Analysis of conceptual connections intermediated by the terminology

\author{
João Batista Ernesto de MORAES (1) y Maura Duarte Moreira GuARIDo (2) \\ (1) Faculdade de Filosofia e Ciências - Unesp, - Av. Higyno Muzzi Filho, 737 Marília - São Paulo - Bra- \\ sil - CEP: 17525-900, jota@marilia.unesp.br (2) mauraguarido@gmail.com
}

\begin{abstract}
Resumen
La Organización del Conocimiento se relaciona con las tareas de clasificar, indexar y representar un conocimiento registrado y socializado. Se trata de una ciencia tridimensional, ya que se ocupa de los principios, métodos e instrumentos utilizados para la gestión del conocimiento humano desde una triple perspectiva: su representación, su organización y su comunicación documental. Así, cuando se aborda la cuestión de la Organización del Conocimiento no se puede prescindir de la Terminología, toda vez que por ésta pasa todo el proceso de aquélla. De esta forma, se objetiva analizar el uso de la Terminología para la identificación y representación de conceptos a partir de las categorías (clases) en los lenguajes documentarios (alfabéticos y jerárquicos). Para eso, se escogió una clase de un sistema decimal y, dentro de esta clase, se comprobó la organización de los conceptos a través de sus relaciones conceptuales (subordinación y coordinación). Como resultado de la investigación, se obtuvo un plano de las relaciones conceptuales, con especial atención a las relaciones de subordinación y coordinación, habiéndo éstas destacado en la representación terminológica de los conceptos analizados. Se puede concluir que el análisis terminológico -considerando su carácter instrumental para el Análisis Documentario, al dedicarse a la atribución de conceptos distintos y metodológicamente delimitados al término- es de especial relevancia para los lenguajes especializados, toda vez que identifica los conceptos de un determinado área del saber para, después, pasar a la atribución de los términos, definiéndolos y controlando sus relaciones.
\end{abstract}

Palabras clave: Lenguajes jerárquicos. Categorías. Conceptos jerárquicos. Terminología.

\section{Introdução}

A Organização do Conhecimento, pela sua importância em sistemas de recuperação de informação, sempre mereceu papel de destaque no ensino e na pesquisa na área da Ciência da Informação.

Desta forma, a Organização do Conhecimento relaciona-se às tarefas de classificar, indexar e representar um conhecimento registrado e soci-

\begin{abstract}
Knowledge Organization is connected with the tasks of classifying, indexing and representing a registered and socialized knowledge. It is, therefore, a threedimensional science, seeing that it is occupied with the principles, methods and instruments used for the management of human knowledge from a triple perspective: its representation, its organization and its documental communication. Thus, when approaching the Knowledge Organization subject, there is no chance to dispense the Terminology, as this one permeates the whole process of the other. In this way, the aim is to analyze the use of Terminology for the identification and representation of concepts from the categories (classes) of documentary languages (alphabetical and hierarchic). In order to achieve such goal, a class of a decimal system was chosen and, inside of this class, the organization of concepts through its conceptual connections (subordination and coordination) was verified. As a result, a detailed inquiry about the conceptual connections was made, with prominence to the subordination and coordination connections, having those achieved notoriety in the terminological representation of the analyzed concepts. It can be concluded that the terminological analysis, considering its instrumental character to the Documentary Analysis-when applied to the attribution of distinguished and methodologically delimited concepts to the terms from their own context-is of special relevance to the Specialized Languages, as it identifies the concepts from a determined area of knowledge and, after that, attributes the terms, defining them and controlling its connections.
\end{abstract}

Keywords: Hierarchic languages. Categories. Hierarchic concepts. Terminology. Terminology.

alizado como forma de atender às necessidades de informação.

Assim, ao se abordar a questão da Organização do Conhecimento não há como prescindir da Terminologia, uma vez que esta permeia todo o processo daquela, desde a fase de classificação até a fase de transmissão do conhecimento.

Desta forma, este estudo teve por objetivo a análise do uso da Terminologia para a identifi- 
cação e representação de conceitos a partir das categorias (classes) nas Linguagens Documentais (Alfabéticas e Hierárquicas).

Para tanto, escolheu-se uma classe de um sistema decimal e, dentro desta classe, verificouse a organização de conceitos através de seus relacionamentos conceituais (subordinação e coordenação).

\section{Organização do conhecimento}

Constituída por num conjunto de leis, princípios e procedimentos pelos quais o conhecimento especializado se estrutura no âmbito de qualquer disciplina, a organização do conhecimento se constrói a partir de uma convergência teórico-metodológica de elementos oriundos da Lingüística, da Documentação, da Informática e da Comunicação, assim como mantém relações efetivas com outras disciplinas que, por sua vez, igualmente se ocupam da produção do pensamento científico, tais como a Filosofia da Ciência, a Sociologia da Ciência.

Pode-se, ainda, caracterizar o surgimento da Organização do Conhecimento como uma confluência de preocupações da das Ciências Cognitivas, das Ciências da Informação e da Comunicação e da Ciência da Computação.

Trata-se, portanto, de uma ciência tridimensional, já que se ocupa dos princípios, métodos e instrumentos utilizados para a gestão do conhecimento humano desde uma tríplice perspectiva: sua representação, sua organização e sua comunicação documental.

O conhecimento, enquanto produto, necessidade e dínamo social, realiza-se a partir da informação (e, ao socializar-se, nela novamente se transforma) e possui uma estrutura e um processo de comunicação abertos. Seu registro e socialização ocorre em documentos (conjunto organizado de dados disponíveis), se expressa por meio de conceitos e tem seu processo de organização operado por meio de sistemas de conceitos, que se organizam mediante sistemas.

Um conceito é, então, uma unidade concentrada de conhecimento. Nenhum conceito pode estar isolado, já que eles constituem entidades abstratas porque sempre são construídos a partir de outros conceitos.

Assim, ao se abordar a questão da Organização do Conhecimento não há como prescindir da Terminologia, uma vez que esta permeia todo o processo daquela, desde a fase de classificação até a fase de transmissão do conhecimento.
A respeito da importância da organização do conhecimento, Barité (2001, p. 39) destaca os seguintes pontos:

- A humanidade só avança na medida em que consegue sistematizar o saber acumulado para cumprir determinados propósitos;

- é preciso estabelecer consensos sobre esse saber, pois, somente dessa maneira, se faz possível o intercâmbio, a comunicação, o debate e a difusão do conhecimento especializado;

- o exposto nos obriga a uma análise exaustiva sobre a natureza, as fontes, os limites e os modos de circulação do conhecimento.

Segundo García Marco (1995, p. 220), a organização do conhecimento é uma disciplina científica (ou seja, sujeita ao método científico) e social, de caráter aplicado.

O conceito de organização do conhecimento é um dos mais antigos com o que tem convivido o homem. A classificação do saber e do conhecimento científico surgiram pela necessidade do homem sistematizar todo o seu conhecimento sobre o mundo exterior e sobre o processo do conhecimento.

Segundo Barité (2001, p. 38), na antiguidade, foram os filósofos que começaram a organizar o conhecimento, em particular Aristóteles, estabelecendo as bases lógicas e epistemológicas que ainda hoje norteiam o pensamento ocidental.

Quando a ciência separou-se da filosofia, outros buscaram sistematizar o saber acumulado da humanidade: primeiro os monges, como guardiões do conhecimento, e depois os primeiros cientistas leigos que provocaram as grandes revoluções intelectuais da historia, a partir de Galileu.

Ainda segundo Barité (2001, p. 39), partir da década de 1950 do século passado, precedidos apenas por Ranganathan, começam a surgir os classificacionistas, especialistas dedicados à criação de instrumentos mais elaboras, como tesauros ou sistemas de classificação mais especializados.

A expressão Organização do Conhecimento (Knowledge Organization - KO, em inglês) foi cunhada por Henry Bliss em uma obra intitulada Organization of Knowledge in Libraries, editado em 1933. Em 1971, Soergel propôs a expressão KO, aceita por vários pesquisadores. Em 1989 é fundada a ISKO (International society for Knowledge Organization). Em 1993, a revista 
International Classification muda seu nome para Knowledge Organization.

A temática Organização do Conhecimento tem se constituído, no decorrer dos últimos anos, em objeto de estudos da Análise Documental, sob a influência da Lingüística e da Lógica, com o intuito de se organizar e recuperar a informação.

A Análise Documental e a Teoria da Classificação sofreram influências teóricas da Organização do Conhecimento, através da International Society of Knowledge (ISKO), nas suas vertentes alemã e espanhola, que deram uma dimensão pedagógica por meio dos estudos de Harmonização Curricular efetuados pelas 43 escolas de Biblioteconomia do Mercosul.

A Organização do Conhecimento passou a ser estudada pelos professores de Análise Documental, e passou-se a dar ênfase ao conhecimento, pois este é expresso em conceitos que se organizam mediante sistemas.

\section{Análise Documental}

Considerando que a Ciência da Informação se articula primordialmente no trinômio produção, organização e uso da informação, tem-se a Análise Documental como um recurso basilar para a organização, já que esta apresenta um conjunto de procedimentos metodológicos notadamente para a definição do conteúdo temático de documentos de modo a permitir a recuperação, o acesso e o uso da informação neles contida.

Especificamente no contexto da organização da informação, insere-se a $A D$ no amplo espectro do denominado tratamento temático, em cujo âmbito constitui a identificação de conceitos uma de suas etapas mais complexas, seja por envolver aspectos subjetivos do analista seja, ainda, pelo fato de a Ciência da Informação vir se dedicando, mais tradicionalmente ao estudo da etapa que a sucede - a representação documental - com ênfase nas linguagens de indexação (Moraes e Guimarães, 2006, p. 120)

Em sentido mais estrito, e considerando que a AD abriga, em seu bojo, e paradoxalmente, como já destacou Guimarães (2003, p. 112), etapas de análise e de síntese, pode-se dizer que os maiores esforços devem centrar-se nos procedimentos metodológicos que visam a desconstruir ou decompor o conteúdo temático do documento para que se possa, assim, posteriormente, reconstruí-lo por meio de representações documentais, como o resumo ou o índice.

A concepção de $A D$ provém, principalmente, dos estudos de Jean-Claude Gardin, a partir de suas experiências nas décadas de 60 e de 70 . Para Gardin (1969) na AD é essencial a linguagem documental para converter o conteúdo dos textos em informação e, para tanto, foi proposto um modelo de linguagem, constituída por um conjunto de termos (o léxico), por relações entre as unidades lexicais, determinadas a priori (o eixo paradigmático) e por uma sintaxe que articula os encadeamentos entre os termos da linguagem, face a um documento específico (o eixo sintagmático (Gardin, 1973).

Nota-se claramente nesta definição a influência de uma das dicotomias saussurianas (Sintagma $X$ Paradigma), o que evidencia, também, que a base das teorias gardinianas sobre $A D$ repousa em teorias lingüísticas.

No Brasil a $A D$ vem sendo objeto de pesquisas desde a década de 1980, notadamente pelos integrantes do Grupo TEMMA da ECA-USP, que vem se dedicando, dentre outros aspectos, à pesquisa sobre as contribuições da Lingüística, da Lógica e da Terminologia à Análise Documental.

Dessa maneira, o grupo vem construindo sua ação em três eixos fundamentais, tal como pode ser observado na ementa disponível no Diretório de Grupos de Pesquisa do CNPq:

A elaboração de diferentes pesquisas permitiu diferenciar claramente, na temática do grupo, 3 eixos de ação: a) o processo da análise documentária, principalmente a indexação, enquanto procedimento de representação da informação contida em documentos e a elaboração de resumos; b) a função comunicacional dos produtos gerados pela análise documentária (resumos e índices); c) a construção de linguagens de organização e transferência de informação. O Grupo TEMMA vem diversificando o escopo de suas pesquisas, investindo particularmente nos estudos de terminologia aplicada à organização e transferência da informação; processos de leitura que sustentam a análise documentária e a reflexão acerca da linguagem de especialidade da Ciência da Informação. As pesquisas, inicialmente limitadas aos documentos textuais, passaram a incluir os documentos audiovisuais, arquivísticos e jurídicos. O Grupo TEMMA é reconhecido nacionalmente como o único grupo formalmente voltado para as pesquisas relacionadas à organização da informação: seus pesquisadores orientam na pós-graduação e atuam na graduação em temáticas relacionadas aos objetivos do Grupo e sua produção bibliográfica constitui bibliografia básica para os cursos de graduação e pós-graduação brasileiros e espanhóis. Intercâmbios vêm se desenvolvendo com pesquisadores portugueses (Universidade do Porto) e espanhóis (Universidade Autônoma de Madri, Salamanca e Carlos III). (grifos nossos)

De uma análise da referida ementa, observa-se que a questão da análise enquanto objeto de 
investigação no intuito de delinear procedimentos constitui a preocupação primeira do grupo, aspecto que encontra respaldo na própria definição de $A D$ proposta por Gardin (GARDIN et al., 1981, p. 29) enquanto "conjunto de procedimentos efetuados com a finalidade de expressar o conteúdo dos documentos científicos, sob formas destinadas a facilitar a recuperação da informação" (Grifos nossos).

$\mathrm{Na}$ definição gardiniana, observam-se, em grifo, os três pontos que embasam sua concepção: o objetivo imediato (a busca por procedimentos), o objetivo mediato (a identificação do conteúdo) e o objeto (documentos científicos).

Desta forma, a Análise Documental de Conteúdo, segundo Pinto e Gálvez (1996, p. 31), é um processo duplo de identificação e representação do conteúdo do texto/documento, processo que transcende as noções convencionais de conteúdo como objeto de estudos, e está extremamente ligado a concepções mais recentes sobre os fenômenos simbólicos, no contexto de uma consciência distinta sobre a comunicação humana, dos novos meios de comunicação, e do papel que estes desempenham na transmissão de informação.

Ainda segundo as autoras, os processos constituem o verdadeiro núcleo de análise. Frente aos conteúdos, objeto quase exclusivo da aprendizagem tradicional, os processos converteram-se na chave do aprendizado significativo.

A série de processos cognitivos parte da sensibilização ou contextualização mental do analista, e termina com a avaliação, passando pela atenção, aquisição, recuperação e transmissão do material estudado.

\section{Sistemas Decimais e as relações conceituais intermediadas pela Terminologia}

Considerando-se o sistema de classificação enquanto estrutura metódica de classes ligadas entre si sobre a base de possessão de uma série de caracteres comuns, observa-se no ato de classificar o processo de inserir o documento em uma classe dentro de um esquema, ou ainda, agrupar um conjunto de documentos a partir de características comuns de conteúdo, de modo a tornar explícitas a subordinação e a coordenação de tais classes.

Deste modo, o conceito de assunto, enquanto representação de conteúdo, não inclui somente uma multiplicidade de referências físico-estruturais das entidades classificadas (com seu correlato na realidade), mas que, por ser produto de uma reflexão humana, cada conceito, e/ou conjunto de todos eles, remete a uma série diversa de elementos tais como: características, funções, processos, operações, propriedades, introduzidos pelo autor do documento e que, por conseguinte, se devem desvelar durante a indexação.

Esses elementos se conhecem como facetas de assunto, baseando-se sua existência no fato de que o conteúdo de um conceito não pode ser compreendido de modo isolado do resto, mas que a plena compreensão de seu significado somente é possível a partir de seu agrupamento com outros assuntos em um mesmo campo semântico e o desvelamento das relações esteja com estas categorias, assim como as que estabelecem o campo ao qual pertence com a totalidade dos campos do conhecimento humano.

Foi pensando nestes agrupamentos de conceitos em seus relacionamentos coordenados e subordinados que se propôs a avaliação dos conceitos de subordinação e coordenação da estrutura hierárquica de sistemas decimais de classificação bibliográficas.

Para tanto, teve-se como objetivos entender a gênese estrutural das categorias analisadas em uma área do conhecimento; fornecer subsídios para se construir hierarquias lógicas no tesauro e subsidiar o ensino de sistemas de classificações.

Ao se analisar uma linguagem documental hierárquica, é necessário conhecer com rigor como se apresenta a ordem hierárquica dos conceitos, e o que significa o princípio da inclusividade.

Estes princípios devem estar presentes tanto na análise quanto na construção de hierarquias conceituais, porque, ao se elaborar uma estrutura hierárquica e suas respectivas relações semânticas, é importante buscar, no assunto escolhido, os diferentes termos que serão utilizados para expressar cada conceito e elegê-lo como termo preferido.

Essa técnica é conhecida como análise por facetas. A análise categorial por faceta proporciona princípios teóricos eficazes para elaborar os processos de organização das árvores conceituais (hierarquização).

Como exemplo, apresenta-se, a seguir, uma hierarquia de conceitos a partir da temática Ensino Superior. Deve-se ressaltar que esta hierarquia foi baseada na classe 378 da estrutura classificatória do Sistema de Classificação Bibliográfico de Dewey. 
378 Educação Superior

Organização e atividade em

Educação Superior

Graus acadêmicos e tópicos relacionados

378.3 Ajuda estudantil e tópicos relacionados

378.4 -.9 Educação Superior em continentes, países, etc., do mundo moderno

O desdobramento a seguir demonstra a divisão, e suas respectivas subdivisões, da classe 378.1

\author{
378.1 Organização e atividade em Educação Su- \\ perior \\ 378.101 Administração de Universidades e Facul- \\ dades \\ 378.[102] (Não pode ser usado) \\ 378.103 Relações Comunitárias \\ 378.104 Cooperação em Ensino Superior \\ 378.[105] (Não pode ser usado) \\ 378.106 Gestão Financeira \\ 378.107 Gestão Executiva
}

Pode-se observar que a adição de um número significa que o termo está num nível hierárquico inferior em relação ao termo que o precede, numa relação todo - parte, estabelecendo-se uma relação de subordinação no sentido crescente, e uma relação de superordenação no sentido decrescente. Neste sentido, 378 é superior a 378.1 e, por sua vez, 378.1 é superior a 378.11 .

Nos sistemas hierárquicos, como os ilustrados acima, os conceitos estão relacionados por suas similitudes. O critério de subordinação determina a estrutura do sistema, e isto acontece entre conceitos de um conjunto, no qual cada conceito é superior ao conceito seguinte, por uma característica normativa (ISO 1087).

Para Dahlberg, apud Campos (1994, p. 107), "Conceito é unidade de conhecimento, pois conhecimento pressupõe um entendimento mais objetivo de algo observável".

Para Barité, nenhum conceito funciona de forma isolada. Ele não é intrinsecamente entidade abstrata isolada, pelo contrário, sempre está construído a partir de outros conceitos, e somente é possível concebê-los em suas relações recíprocas, e em seu maior ou menor parentesco como outras noções. As classificações científicas, as taxionomias, e as nomenclaturas são os sistemas de conceitos mais usados, dentro das diferentes especialidades, e os que, tradicionalmente, servem de modelo de organização aos sistemas de classificação e aos tesauros. (Barité, 2001, p. 52).

Dentro destes sistemas de classificação, mais notadamente o de classificação decimal, pode- se observar um arranjo de subordinação lógica (relação vertical). O conceito subordinado (espécie) possui uma característica a mais que o conceito imediatamente superior (gênero). A transição do todo/parte é também conhecida como relação partitiva e possui vários pontos comuns com a relação genérica, de forma que a distinção é, às vezes, sutil e praticamente se confundem, tantos nos sistemas de classificação, como nos tesauros, sob o termo hierárquico.

Desta forma, segundo Campos (1994, p. 37), as relações genéricas "podem ser entendidas como conceitos superordenados que comportam as mesmas características das noções subordinadas a partir delas, além do que ocupam um lugar fundamental no tesauro."

Na visão de Cintra et al. (2002, p. 51), o sistema nocional de uma área do conhecimento constitui-se em arcabouço fundamental para a construção da Linguagem Documental, na medida em que possibilita a materialização das relações entre as noções. As autoras mostram que, conforme a norma ISO 1087 (Cintra et al. 2002, p. 51), "um sistema nocional define-se como um conjunto estruturado de noções que reflete as relações estabelecidas entre as noções que o compõem e no qual cada noção é determinada pela sua posição no sistema." As noções expressas por meio dos termos e símbolos, são influenciadas pelo contexto sócio-cultural. Desse modo, segundo a ISO 1087 (1990) a noção ou conceito, por sua vez, define-se como "unidade de pensamento constituído por propriedades comuns a uma classe de objetos."

Uma linguagem focada em um contexto, ou seja, em uma área especializada, naturalmente elimina as possíveis diferenças de significado de uma palavra. Para Cintra et al. (2002, p. 71), "não podem existir, por exemplo, duas ou mais palavras que se refiram a um mesmo conceito ou uma palavra para designar vários conceitos."

Nesse sentido, destacam-se alguns fenômenos da Lingüística, reconhecidos pela Linguagem Documental no controle de vocabulário para tratamento e recuperação da informação: a polissemia, que decorre ou da organização sintático-semântica, com a finalidade de distinguir a plurissignificação como fenômeno geral; ou da ambigüidade, que ocorre quando há mais de uma interpretação em função da plurissignificação, que como a polissemia, poderá também levar a ambigüidade (Cintra et al., 2002, p. 7172).

Em Sistemas de Informação a plurissignificação não é permitida, porque a presença de termos com vários significados, ou várias interpretações 
contribui para o aumento do ruído na recuperação das informações, reduzindo o nível de eficiência do sistema. Assim, Cintra et al. (2002, p. 72) afirmam que "numa linguagem documentária, tanto a polissemia quanto à ambigüidade devem ser neutralizadas, para que seja garantida a monossemia".

Sinonímia, de acordo com a norma ISO 1087 (1990) é a relação entre designações de uma mesma língua que representam o mesmo conceito. Conforme Lara (1999, p. 105), "os sinônimos nas linguagens documentais permitem estabelecer as relações de equivalência entre termos preferidos e não-preferidos, funcionando como meio de controle da variação de significado e como recurso para o acesso à linguagem do sistema".

Hiponímia, segundo Lyons (1976, p. 308), é "a relação existente entre um lexema mais específico ou subordinado e um lexema mais geral ou superordenado". O autor ainda explica que hiponímia refere-se aos "termos mais específicos abrangidos por um termo mais geral [...], por exemplo: rosa, tulipa, são hipônimos de flor". Por hiponímia quer-se dizer a inclusão do significado de um termo no significado de outro, em outras palavras, pode ser uma relação espécie/gênero que constitui uma classe.

Por esse motivo, no percurso de construção de uma Linguagem Documental que compreende o controle de vocabulário e, conseqüentemente, o controle dos termos e as relações entre eles, abordam-se freqüentemente questões ligadas ao tratamento terminológico.

Nesse sentido, a Terminologia, com suas regras e conceitos, contribui para que esse processo ocorra de forma precisa, uma vez que as Linguagens Documentais são elaboradas em áreas específicas para atender usuários com interesses específicos, assim, os termos pertencem ao domínio comum dos usuários que a utilizam.

$\mathrm{Na}$ metodologia de construção de Linguagem Documental e na Terminologia encontram-se muitas definições que se assemelham e percebe-se convergência nos aspectos metodológicos utilizados para a organização do conhecimento.

A Terminologia, segundo Benveniste (1989, p. 252), preocupa-se em estudar a conceituação e a identificação dos signos e os termos apropriados para o contexto. A Linguagem Documental, enquanto instrumento de mediação, preocupase com a terminologia adotada para o assunto e se está adequada ao contexto, para assegurar a eficácia da recuperação da informação para usuários de uma especialidade, porque a rela- ção hierárquica dos sistemas decimais baseiase no critério de inclusividade, formalizado na teoria de conjuntos, e gerando uma relação de ordem no conjunto de conceitos para o qual todo conceito está incluído em outro conceito de nível superior, incluindo, obviamente, o que dá nome ao conjunto (conceito maior), que pertence a uma ordem superior.

$\mathrm{Na}$ lógica clássica, podem-se encontrar dois métodos para definir um conceito: o conceito maior (hierarquicamente superior), que inclui em sua intensão a do conceito subordinado, assim como todos os outros relacionados que formam sua abrangência (García Marco, 1996, p. 179).

\section{A compreensão dos Sistemas Decimais através de Mapas Conceituais}

Para Ausubel (1989) "a assimilação é o ponto central da aprendizagem significativa". Mediante o processo de assimilação (mapa conceitual) toda "nova informação está vinculada aos aspectos relevantes e pré-existentes da estrutura cognitiva", e como resultado dele tanto a informação adquirida como a própria estrutura cognitiva relacionam-se.

A aprendizagem consiste, neste ponto de vista, em uma mudança das estruturas cognitivas que se pode produzir por três vias, segundo Garcia Marco (1996, p. 180):

Aprendizagem subordinada que se produz mediante a diferenciação progressiva dos conceitos existentes em vários outros conceitos de nível inferior". Com isso a estrutura cognitiva ganha em potência analítica e precisão.

A aprendizagem superordenada que se produz de baixo para cima mediante a conciliação integradora de vários conceitos em um nível superior". Portanto, a estrutura cognitiva consegue maior integração porque os conceitos mais gerais (extensão) permitem uma maior economia cognitiva e uma navegação mais eficaz pelo mapa conceitual.

Aprendizagem combinatória pela qual se constrói uma nova relação entre dois ou mais conceitos sem que se produza inclusão de alguns conceitos em outros.

Pinto y Gálvez (1996, p. 75) definem mapa conceitual como "versão sofisticada do mapa semântico que supõe uma maneira de representar graficamente os conceitos os conceitos e relações entre eles que intervêm na unidade textual". 


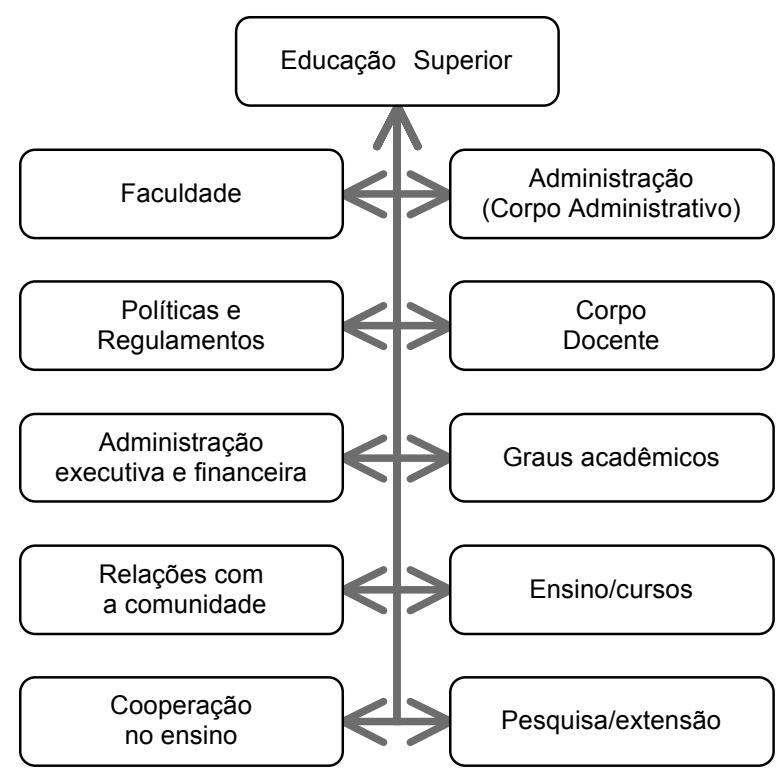

Figura 1. Educação Superior

Como pode ser visto na figura 1 , foi construída uma estrutura hierárquica retirada da classe 378 - Educação Superior da CDD (Classificação Decimal de Dewey), 22.ed, 2003, na qual os conceitos foram identificados e selecionados, partindo-se dos mais importantes, e ordenados hierarquicamente. As palavras de ligação foram construídas com o objetivo de formar proposições que se relacionam através das linhas do mapa.

\section{Considerações finais}

A técnica do mapa conceitual é um dos pontos da aprendizagem significativa que tem norteado o ensino das disciplinas que se ocupam dos processos de comunicação das unidades de informação e documentação (análise e linguagens documentais).

Esta técnica advém da proximidade dos domínios da investigação em suas bases teóricas, a qual abre todo um campo interdisciplinar, especialmente a Lingüística e a Lógica.

Pode-se concluir que a análise terminológica, considerando-se seu caráter instrumental para a Análise Documental, ao dedicar-se à atribuição de conceitos distintos e metodologicamente delimitados ao termo, a partir do contexto do mesmo, é de especial relevância às Linguagens Especializadas, uma vez que identifica os conceitos de uma determinada área do saber para, em seguida, passar-se à atribuição dos termos, definindo-os e controlando suas relações de sinonímia, homonímia, etc., o que corrobora uma assertiva já levantada pelo Grupo TEMMA quanto à importância da Terminologia no processo de representação; indo além, destaca-se a importância no processo de análise documental.

Tem-se como recomendação a aplicação destes dados no ensino de Análise Documentária, com vistas a uma flexibilização no momento da representação, permitindo maior agilidade na incorporação de novos conceitos, valendo-se do apoio teórico da Terminologia em áreas especializadas.

\section{Notas}

(1) O referido grupo, instituído no início dos anos 80 por Johanna Smit, que introduziu no país os estudos de análise documental a partir da matriz gardiniana, construiu uma tradição de pesquisa especificamente na área de análise documental, centrando seu enfoque nos aspectos lingüísticos, lógicos e terminológicos que incidem sobre a análise documental. Atualmente, o grupo conta com os seguintes pesquisadores: Anna Maria Marques Cintra, João Batista Ernesto de Moraes, Johanna Wilhelmina Smit, José Augusto Chaves Guimarães, Maria de Fátima Gonçalves Moreira Tálamo, Mariângela Spotti Lopes Fujita, Marilda Lopes Ginez de Lara, Nair Yumiko Kobashi, Vânia Mara Alves Lima.

\section{Referências}

Ausubel, D. P. (1989). Psicología educativa: un punto de vista cognoscitivo. México: Trillas, 1989.

Barité, M. (2001). Organización del conocimiento: un nuevo marco teórico - conceptual en bibliotecología y documentación. // Carrara, K. Educação, universidade e pesquisa. Marília: Fapesp, 2001. 35-60.

Benveniste. É. (1989). Problemas de lingüística geral II. Campinas: Pontes, 1989.

Campos, M. L. de A. (1994). Em busca de princípios comuns na área de representação da informação: uma comparação entre o método de classificação facetada, o método do tesauro-baseado-em-conceito e a teoria geral da terminologia. Universidade Federal do Rio de Janeiro, 1994. Dissertação de Mestrado em Ciência da Informação.

Cintra, A. M. M.; et. al. (2002). Para entender as linguagens documentárias. São Paulo: Polis, 2002.

Dewey, M. (2003). Dewey decimal classification and relative index. 22.ed. Dublin: OCLC, 2003.

García Marco, F. J. (1995). Los contenidos y la secuencia docente de la Organización y Representación del Conocimiento: una propuesta interdisciplinar. // Scire. 1:1 (1995) 219-28.

García Marco, F. J. (1996). Implicaciones teóricas y didácticas de la técnica de los mapas conceptuales para enseñanza de contenido y de los lenguajes documentales. // Scire. 2:2 (1996) 175-186.

Gardin, J.-C. (1969). Semantic analysis procedures in the sciences of man. Social Science Information. 8:1 (1969) 17-42.

Gardin, J.-C. (1973). Document analysis and linguistics. Journal of Documentacion, London. 29:2 (1973) 137168. 
Gardin, J.-C. et alii. (1981). La logique du plausible: essais d'épistemologie pratique. Paris: Ed. Maison des Sciences de l'Homme, 1981.

Guimarães, J. A. C. (2000). Condensação documentária em legislação e jurisprudência: elementos instrumentais para a construção de ementas. Marília, 2000. Tese (Livre Docência em Ciência da Informação). Marília: Faculdade de Filosofia e Ciências, Universidade Estadual Paulista,, 2000.

Guimarães, J. A. C. (2003). A análise documentária no âmbito do tratamento da informação: elementos históri$\cos$ e conceituais. // Rodrigues, G. M.; Lopes, I. L. (Org.). Organização e representação do conhecimento na perspectiva da Ciência da Informação. Brasília: Thesaurus, 2003. 100-118.

Lara, M. L. G. de. (1999). Representação e linguagens documentárias: bases teórico-metodológicas. Escola de Comunicações e Artes, Universidade de São Paulo, 1999. Tese de Doutoramento.

Lyons, J. (1976). Novos horizontes em lingüística. São Paulo: Cultrix, 1976.

Moraes, J. B. E.; Guimarães, J.A.C. (2006). Análisis documental de contenido de textos literarios narrativos: en busca del diálogo entre las concepciones de aboutness/meaning y de recorrido temático/recorrido figurativo. // Scire. 12 (2006) 120-135.

Pinto, M.; Gálvez, C. (1996). Análisis documental de contenido: procesamiento de información. Madrid: Editorial Sintesis, 1996. 